\title{
General validity of reciprocity in quantum mechanics
}

\author{
H. Y. Xie (謝懷毅), P. T. Leung (梁培德), ${ }^{*}$ and D. P. Tsai (蔡定平) \\ Department of Physics, National Taiwan University, Taipei 10617, Taiwan, Republic of China
}

(Received 9 October 2008; published 15 December 2008)

\begin{abstract}
The concept of reciprocity symmetry for matter-wave propagation is established for nonrelativistic quantum mechanics with previous results in the literature extended to include nonlocal interactions. Examples are given for cases with both local and nonlocal potentials, where we show in particular that reciprocity can be violated for the motion of a charged particle in an external electromagnetic field. In addition, this symmetry is applied to interpret a recent analysis [Phys. Rev. A 64, 042716 (2001)] on the symmetry of transmission through one-dimensional complex potentials, with the emphasis that the validity of reciprocity can go beyond that of time-reversal symmetry, such as in the presence of absorption in which the latter symmetry breaks down.
\end{abstract}

DOI: 10.1103/PhysRevA.78.064101

PACS number(s): 03.65.-w, 41.20.Jb

\section{INTRODUCTION}

In any wave propagation from a source to a receiver (detector) with both fixed at separate locations, the wave can undergo various scattering processes via interaction with targets in the environment. In such a process, reciprocity symmetry refers to the equality in the signal received when the source and detector are reversed (i.e., with their respective positions switched) $[1,2]$. This is a rather powerful symmetry which finds many interesting applications based on either its validity or its breakdown, in a large number of areas involving transmission of signals ranging from classical optics [1] to quantum-mechanical scattering problems [2]. Hence the understanding of the applicability and limitation of this symmetry principle is of high significance in any domain of wave physics.

Mathematically, while this principle can be expressed in different forms [1], one of the most general and unified ways is to refer to the symmetry of the Green's function for the associated wave equation. This is so because of the fact that any signal transmitted can be expressed as an integral over the product of the appropriate Green's function and the source [e.g., in electrodynamics we have the vector potential expressed as $\left.\vec{A}(\vec{r}) \sim \int G\left(\vec{r}, \vec{r}^{\prime}\right) \vec{J}\left(\vec{r}^{\prime}\right) d^{3} \vec{r}^{\prime}\right]$. In optics, while the study of reciprocity has a long history starting from Stokes' study on transmission and reflection symmetry and the publication of the Lorentz lemma more than a century ago [1], recent revival in the interest of this principle has focused on the wave propagation through various complex media such as photonic crystals in which both anisotropic and nonlocal electrodynamic responses may be involved [3-5]. In particular, we have recently clarified that within linear optics, reciprocity can be invalidated only by certain failure of symmetry conditions in the dielectric tensors, but otherwise remains valid even in the presence of dissipation and nonlocal response from the medium involved [4,5].

In quantum mechanics (QM), however, this symmetry seems to have received less attention in the literature. Except

\footnotetext{
*Corresponding author. Also at Institute of Optoelectronic Sciences, National Taiwan Ocean University, Keelung, Taiwan, R.O.C., and Department of Physics, Portland State University, P.O. Box 751, Portland, Oregon 97207-0751, USA.
}

for the very nice work of Bilhorn et al. many years ago [2], which had clarified the concepts of reciprocity, time-reversal symmetry, and the Hermiticity of the Hamiltonian in one context, the importance of this symmetry seems to have been somewhat overlooked over the years [6]. For example, a recent study on the symmetry between the left and right incidence of the projectile in a one-dimensional (1D) quantum (complex) potential scattering problem seemed to have completely ignored the role of reciprocity underlying all the results as obtained in the author's work [7]. The purpose of this Brief Report is to reemphasize the importance of this symmetry principle [2] in the understanding of various phenomena involved in 1D QM scattering [7] and to generalize the previous work [2] to cover the case when a nonlocal interaction is involved. We shall limit ourselves to problems with nonrelativistic potential scattering.

\section{THEORY}

We start by considering the following stationary Schrödinger equation:

$$
-\frac{\hbar^{2}}{2 m} \nabla^{2} \psi(\vec{r})+\int V\left(\vec{r}, \vec{r}^{\prime}\right) \psi\left(\vec{r}^{\prime}\right) d^{3} \vec{r}^{\prime}=E \psi(\vec{r}),
$$

where we have introduced a nonlocal interaction potential which is also allowed to be generally complex. This kind of model finds application in many realistic physical problems, notably in nuclear physics where it is known as the velocitydependent nonlocal optical potential [8]. Let us now introduce the Green's function of the problem defined via

$$
\begin{aligned}
& -\frac{\hbar^{2}}{2 m} \nabla^{2} G\left(\vec{r}, \vec{r}^{\prime}\right)+\int V\left(\vec{r}, \vec{r}_{1}\right) G\left(\vec{r}_{1}, \vec{r}^{\prime}\right) d^{3} \vec{r}_{1}-E G\left(\vec{r}, \vec{r}^{\prime}\right) \\
& =\delta\left(\vec{r}-\vec{r}^{\prime}\right) .
\end{aligned}
$$

We want to establish the conditions for the symmetry of the Green's function: i.e.,

$$
G\left(\vec{r}, \vec{r}^{\prime}\right)=G\left(\vec{r}^{\prime}, \vec{r}\right) .
$$

Note that we will use the symbols $\vec{\nabla}, \vec{\nabla}^{\prime}, \ldots$ to denote differentiation operators with respect to the coordinates $\vec{r}, \vec{r}^{\prime}, \ldots$ In addition, the Green's function we consider here is very gen- 
eral and may not observe translational invariance in the coordinates except for some special potential functions. To establish the conditions for (3) to be valid, we consider a similar equation for $G\left(\vec{r}, \vec{r}^{\prime \prime}\right)$ as follows:

$$
\begin{aligned}
& -\frac{\hbar^{2}}{2 m} \nabla^{2} G\left(\vec{r}, \vec{r}^{\prime}\right)+\int V\left(\vec{r}, \vec{r}_{1}\right) G\left(\vec{r}_{1}, \vec{r}^{\prime}\right) d^{3} \vec{r}_{1}-E G\left(\vec{r}, \vec{r}^{\prime}\right) \\
& \quad=\delta\left(\vec{r}-\vec{r}^{\prime \prime}\right) .
\end{aligned}
$$

Following similar steps as in our previous works on optical reciprocity [5], we multiply (2) by $G\left(\vec{r}, \vec{r}^{\prime \prime}\right)$ and (4) by $G\left(\vec{r}, \vec{r}^{\prime}\right)$, taking the difference of the result leads to

$$
\begin{aligned}
& -\frac{\hbar^{2}}{2 m}\left[G\left(\vec{r}, \vec{r}^{\prime}\right) \nabla^{2} G\left(\vec{r}, \vec{r}^{\prime}\right)-G\left(\vec{r}, \vec{r}^{\prime}\right) \nabla^{2} G\left(\vec{r}, \vec{r}^{\prime}\right)\right] \\
& =-\int d^{3} \vec{r}_{1}\left[G\left(\vec{r}, \vec{r}^{\prime}\right) V\left(\vec{r}, \vec{r}_{1}\right) G\left(\vec{r}_{1}, \vec{r}^{\prime}\right)\right. \\
& \left.\quad-G\left(\vec{r}, \vec{r}^{\prime}\right) V\left(\vec{r}, \vec{r}_{1}\right) G\left(\vec{r}_{1}, \vec{r}^{\prime}\right)\right]+G\left(\vec{r}, \vec{r}^{\prime}\right) \delta\left(\vec{r}-\vec{r}^{\prime}\right) \\
& -G\left(\vec{r}, \vec{r}^{\prime}\right) \delta\left(\vec{r}-\vec{r}^{\prime}\right) .
\end{aligned}
$$

Next we integrate over the coordinates $\vec{r}$ and rearrange the result to obtain

$$
\begin{aligned}
G\left(\vec{r}^{\prime}, \vec{r}^{\prime}\right)-G\left(\vec{r}^{\prime \prime}, \vec{r}^{\prime}\right)= & -\frac{\hbar^{2}}{2 m} \int d^{3} \vec{r}\left[\left(\vec{r}, \vec{r}^{\prime}\right) \nabla^{2} G\left(\vec{r}, \vec{r}^{\prime}\right)\right. \\
& \left.-G\left(\vec{r}, \vec{r}^{\prime}\right) \nabla^{2} G\left(\vec{r}, \vec{r}^{\prime}\right)\right] \\
& +\int d^{3} \vec{r} \int d^{3} \vec{r}_{1}\left\{V\left(\vec{r}, \vec{r}_{1}\right)\right. \\
& \times\left[G\left(\vec{r}, \vec{r}^{\prime}\right) G\left(\vec{r}_{1}, \vec{r}^{\prime}\right)\right. \\
& \left.\left.-G\left(\vec{r}, \vec{r}^{\prime}\right) G\left(\vec{r}_{1}, \vec{r}^{\prime}\right)\right]\right\} .
\end{aligned}
$$

The first volume integral on the right-hand side can be converted to a surface integral using Green's theorem and made to vanish by assuming appropriate boundary conditions on $S$ $[2]$ :

$$
\begin{aligned}
G\left(\vec{r}^{\prime}, \vec{r}^{\prime}\right)-G\left(\vec{r}^{\prime \prime}, \vec{r}^{\prime}\right)= & -\frac{\hbar^{2}}{2 m} \oint_{S} d a\left\{\hat { n } \cdot \left[G\left(\vec{r}, \vec{r}^{\prime}\right) \vec{\nabla} G\left(\vec{r}, \vec{r}^{\prime}\right)\right.\right. \\
& \left.\left.-G\left(\vec{r}, \vec{r}^{\prime}\right) \vec{\nabla} G\left(\vec{r}, \vec{r}^{\prime}\right)\right]\right\} \\
& +\int d^{3} \vec{r} \int d^{3} \vec{r}_{1}\left\{V\left(\vec{r}, \vec{r}_{1}\right)\right. \\
& \left.\times\left[G\left(\vec{r}, \vec{r}^{\prime \prime}\right) G\left(\vec{r}_{1}, \vec{r}^{\prime}\right)-G\left(\vec{r}, \vec{r}^{\prime}\right) G\left(\vec{r}_{1}, \vec{r}^{\prime \prime}\right)\right]\right\} \\
= & \int d^{3} \vec{r} \int d^{3} \vec{r}_{1}\left\{V ( \vec { r } , \vec { r } _ { 1 } ) \left[G\left(\vec{r}, \vec{r}^{\prime}\right) G\left(\vec{r}_{1}, \vec{r}^{\prime}\right)\right.\right. \\
& \left.\left.-G\left(\vec{r}, \vec{r}^{\prime}\right) G\left(\vec{r}_{1}, \vec{r}^{\prime}\right)\right]\right\} .
\end{aligned}
$$

Hence by imposing the following symmetry for the potential,

$$
V\left(\vec{r}, \vec{r}_{1}\right)=V\left(\vec{r}_{1}, \vec{r}\right)
$$

the integrand in the last double integral will be antisymmetric in $\vec{r} \leftrightarrow \vec{r}_{1}$ and the integral simply vanishes, thus establishing the result claimed in Eq. (3). Note that the condition in (8) is the same sufficient condition for the orthogonality of the eigenfunctions of the system as shown by Brown and de Dominicis [8].

\section{APPLICATIONS}

One of the most interesting applications of the reciprocity theorem in quantum mechanics is the prediction of the "leftright symmetry" in the transmission coefficients of a $1 \mathrm{D}$ potential scattering problem as observed recently in an explicit calculation [7]. It is important to distinguish this with the time-reversal symmetry by noting that the validity of Eq. (3) is unaffected even with complex potentials for which timereversal symmetry breaks down in the presence of absorption $[1,2]$. On the other hand, in the case of a nonlocal interaction, reciprocity can fail if the symmetry for the potential in Eq. (8) is not fulfilled-even time reversal may remain valid in the case of real potentials. We shall illustrate these features with the following examples.

\section{A. Local potentials}

Let us consider a particle of energy $E$ scattered by a 1D local potential function $V(x)$ which can be arbitrary (i.e., asymmetric about $x=0$ ) and complex. This problem has been studied recently for the symmetry between the scattering of a particle incident from either the left or right side of the potential [7]. For this case, the condition in Eq. (3) is guaranteed to hold [2], which leads immediately to the assurance of the symmetry between the transmission coefficients for the particle to be incident from either side of this arbitrary complex potential. Such an intimate connection between reciprocity and transmission symmetry has been noted previously in the literature of optics $[4,9]$. Note that although in the explicit calculations of [7] plane waves are used for the incoming and outgoing particles, the symmetry in transmission established here in our approach using reciprocity is more general, which goes beyond the plane-wave approximations for the particles. Furthermore, our result also predicts the "left-right symmetry" for the tunneling coefficients through a general asymmetric complex potential in case the energy of the particle is below the "barrier height." Although, in principle, reciprocity does not imply any symmetry for the reflection coefficients, conservation of probability will imply such symmetry in the case of a real potential [10]. However, when the potential is asymmetric and complex, the asymmetric absorptions at different sides of the potential will imply asymmetric reflections for the two cases, leaving only transmission to remain symmetric for incidence from either side because of the condition in Eq. (3). We emphasize again that time-reversal symmetry is not essential for this transmission symmetry since it actually breaks down for complex potentials. Thus we see that the simple application of the reciprocity principle explains all the results concluded in the previous work [7] without any tedious explicit calculations as carried out there.

\section{B. Nonlocal potentials}

To illustrate reciprocity in the presence of nonlocal interactions, we cite the following examples. 


\section{Potentials satisfying Eq. (8)}

There exists a large class of nonlocal optical potentials in nuclear physics which satisfy the symmetry condition in Eq. (8). A well-known example can be found from that suggested by Perey and Buck [11]:

$$
V\left(\vec{r}, \vec{r}^{\prime}\right)=U\left(\frac{\left|\vec{r}+\vec{r}^{\prime}\right|}{2}\right) \frac{\exp \left[-\left(\frac{\vec{r}-\vec{r}^{\prime}}{\beta}\right)^{2}\right]}{\pi^{3 / 2} \beta^{3}},
$$

where $U\left(\frac{\left|\vec{r}+\vec{r}^{\prime}\right|}{2}\right)$ is a Saxon-Woods-type function and $\beta$ a Gaussian width parameter. Hence reciprocity symmetry can be applied to establish the symmetry for the reaction matrix of this potential [2]. For the $1 \mathrm{D}$ case, the nonlocal problem has also been studied in the literature. For example, the potential $V\left(x, x^{\prime}\right)=V_{0}\left(x-x^{\prime}\right)^{2} e^{-\left(x-x^{\prime}\right)^{2} / \beta^{2}}$ has been studied by Baseia for the phase transition between a localized and an extended state [12]. For a problem like this, it will be highly nontrivial to calculate explicitly the various transmission and reflection coefficients to demonstrate the "left-right symmetry." However, such symmetry is guaranteed according to our result from reciprocity symmetry for this potential which satisfies the condition in Eq. (8).

\section{Potentials violating Eq. (8)}

In the literature of electron-atom scattering, asymmetric nonlocal potential has been introduced via the polarized orbital method based on the Hartree-Fock formalism in which $V\left(\vec{r}, \vec{r}^{\prime}\right) \neq V\left(\vec{r}^{\prime}, \vec{r}\right)$ in Eq. (1) [13]. Here we consider a more common example of a velocity-dependent potential with an electron moving in an external electromagnetic field as described by the following Schrödinger equation:

$$
-\frac{\hbar^{2}}{2 m} \nabla^{2} \psi+\left(\frac{e^{2}}{2 m} A^{2}-\frac{i e \hbar}{m} \vec{A} \cdot \vec{\nabla}-e \phi\right) \psi=E \psi,
$$

where $\vec{A}$ and $\phi$ are the vector and scalar potentials, respectively. We want to show that Eq. (10) can be put into a form as in Eq. (1) with a kernel in the nonlocal interaction which is not necessary symmetric: i.e., $V\left(\vec{r}, \vec{r}^{\prime}\right) \neq V\left(\vec{r}^{\prime}, \vec{r}\right)$. To show this, let us first introduce explicitly the "local part" of the potential in Eq. (1) via a $\delta$ function as follows:

$$
\begin{aligned}
\int V\left(\vec{r}, \vec{r}^{\prime}\right) \psi\left(\vec{r}^{\prime}\right) d^{3} \vec{r}^{\prime} & =\int\left[U\left(\vec{r}^{\prime}\right) \delta\left(\vec{r}-\vec{r}^{\prime}\right)+W\left(\vec{r}, \vec{r}^{\prime}\right)\right] \psi\left(\vec{r}^{\prime}\right) d^{3} \vec{r}^{\prime} \\
& =U(\vec{r}) \psi(\vec{r})+\int W\left(\vec{r}, \vec{r}^{\prime}\right) \psi\left(\vec{r}^{\prime}\right) d^{3} \vec{r}^{\prime} .
\end{aligned}
$$

Next, we make change of variable, $\vec{r}^{\prime}=\vec{r}+\vec{a}$, and perform a Taylor expansion of the wave function in the nonlocal integral to obtain

$$
\begin{aligned}
\int W\left(\vec{r}, \vec{r}^{\prime}\right) \psi\left(\vec{r}^{\prime}\right) d^{3} \vec{r}^{\prime}= & \int W(\vec{r}, \vec{r}+\vec{a}) \psi(\vec{r}+\vec{a}) d^{3} \vec{a} \\
= & \int W(\vec{r}, \vec{r}+\vec{a})[\psi(\vec{r})+\vec{a} \cdot \vec{\nabla} \psi(\vec{r}) \\
& \left.+\frac{(\vec{a} \cdot \vec{\nabla})^{2}}{2} \psi(\vec{r})+O\left(a^{3}\right)\right] d^{3} \vec{a}
\end{aligned}
$$

Hence, for potentials of weak nonlocality, where $W(\vec{r}, \vec{r}$ $+\vec{a}) \neq 0$ only for $\vec{a}$ within a small neighborhood about $\vec{r}$, higher-order terms in Eq. (12) can be ignored [3] and we recover the well-known equivalence between a nonlocal and a velocity-dependent potential (through the gradient term $\vec{\nabla} \psi \sim \vec{p} \psi \sim \vec{v} \psi)[8]:$

$$
\begin{aligned}
\int W\left(\vec{r}, \vec{r}^{\prime}\right) \psi\left(\vec{r}^{\prime}\right) d^{3} \vec{r}^{\prime} \approx & \psi(\vec{r}) \int W(\vec{r}, \vec{r}+\vec{a}) d^{3} \vec{a} \\
& +\vec{\nabla} \psi(\vec{r}) \cdot \int W(\vec{r}, \vec{r}+\vec{a}) \vec{a} d^{3} \vec{a} .
\end{aligned}
$$

Hence the Hamiltonian in Eq. (10) can be put into the nonlocal form with local part

$$
\frac{e^{2}}{2 m} A^{2}-e \phi \rightarrow U(\vec{r})+\int W(\vec{r}, \vec{r}+\vec{a}) d^{3} \vec{a}
$$

and nonlocal part

$$
\vec{A}(\vec{r}) \rightarrow-\frac{m}{i e \hbar} \int W(\vec{r}, \vec{r}+\vec{a}) \vec{a} d^{3} \vec{a} .
$$

Next, we show that the potential function $W$ so defined in Eq. (15) is not necessary symmetric in its arguments. Let $\vec{x}$ $\equiv \vec{r}$ and $\vec{y} \equiv \vec{r}+\vec{a}$; then, the integral

$$
\begin{aligned}
\int \vec{A}(\vec{r}) d^{3} \vec{r} & \sim \int W(\vec{r}, \vec{r}+\vec{a}) \vec{a} d^{3} \vec{a} d^{3} \vec{r} \\
& =\int W(\vec{x}, \vec{y})(\vec{y}-\vec{x}) d^{3} \vec{y} d^{3} \vec{x}=0
\end{aligned}
$$

if $W(\vec{x}, \vec{y})=W(\vec{y}, \vec{x})$. But since the integral of the vector potential over all space does not necessary vanish except for special cases such as when rotational invariance is valid [14], we conclude that the kernel $W$ in the integral for $\vec{A}(\vec{r})$ in Eq. (15) is not necessary symmetric in its arguments. Hence, based on the result we proved in the previous section, we conclude that the reciprocity symmetry is not necessary valid for the motion of a quantum particle in an external electromagnetic field [15].

\section{CONCLUSION}

In this work, we have established the condition for reciprocity to be valid for quantum systems involving nonlocal interactions, extending results previously published in the literature [2]. In particular, we have shown that reciprocity is 
not guaranteed and can fail for velocity-dependent potentials, such as that involved in the motion of a charged particle interacting with an external electromagnetic field. Furthermore, we have pointed out that the $1 \mathrm{D}$ transmission symmetry reported in a recent publication can be trivially understood by referring to reciprocity symmetry-without all those tedious calculations as performed in Ref. [7]. It would be of interest to find more interesting applications of this symmetry to other problems, especially to those when nonlocal potentials are involved.

\section{ACKNOWLEDGMENTS}

The authors are grateful to Professor Railing Chang for discussions and to the National Science Council of Taiwan, R.O.C., for research support under Projects No. NSC-972120-M-002-013, No. NSC-96-2923-M-002-002-MY3, and No. NSC 97-2811-M-002-018, respectively, and to National Taiwan University and National Center for Theoretical Sciences, Taipei Office. P.T.L. would like to thank the additional financial support from the Fulbright Foundation.
[1] R. J. Potton, Rep. Prog. Phys. 67, 717 (2004).

[2] D. E. Bilhorn, L. L. Foldy, R. M. Thaler, W. Tobocman, and V. A. Madsen, J. Math. Phys. 5, 435 (1964).

[3] A. Malinowski, Yu P. Svirko, and N. I. Zheludev, J. Opt. Soc. Am. B 13, 1641 (1996).

[4] M. Iwanaga, A. S. Vengurlekar, T. Hatano, and T. Ishihara, Am. J. Phys. 75, 899 (2007).

[5] P. T. Leung and R. Chang, J. Opt. A, Pure Appl. Opt. 10, 075201 (2008); H. Y. Xie, P. T. Leung, and D. P. Tsai, J. Phys. A (to be published).

[6] See also G. Dillon and G. Passatore, Nucl. Phys. A 114, 623 (1968); R. Carminati et al., Phys. Rev. A 62, 012712 (2000).

[7] Z. Ahmed, Phys. Rev. A 64, 042716 (2001).

[8] P. J. Wyatt, J. G. Wills, and A. E. S. Green, Phys. Rev. 119, 1031 (1960); H. Fiedeldey et al., Phys. Rev. A 32, 3095 (1985); G. E. Brown and C. T. de Dominicis, Proc. Phys. Soc. 72, 70 (1958).

[9] J. Lekner, Theory of Reflection of Electromagnetic and Particle Waves (Nijhoff, Dordrecht, 1987).

[10] Note that in optics, generalized reciprocity relations have been derived among transmission, reflection, and absorption. See G.
S. Agarwal and S. D. Gupta, Opt. Lett. 27, 1205 (2002). It is straightforward to establish the analogous results in quantum mechanics.

[11] F. Perery and B. Buck, Nucl. Phys. 32, 353 (1962).

[12] B. Baseia, Phys. Rev. A 38, 1632 (1988).

[13] A. Temkin, Phys. Rev. 107, 1004 (1957); S. R. Singh and A. D. Stauffer, J. Phys. B 7, 782 (1974).

[14] Note that one must be careful in the argument for the integral of the vector potential over all space being not necessary vanishing. One way to see this is to note that $\vec{A}$ does not transform as vector under Galileo (nonrelativistic) transformations [see, e.g., J. D. Bjorken and S. D. Drell, Relativistic Quantum Fields (McGraw-Hill, New York, 1965)]. Another argument is to resort to the "gauge nonuniqueness" of $\vec{A}$. In such a case, one can easily show that even when we have $\int \vec{A} d^{3} \vec{r}=0$, we can transform to another $\vec{A}^{\prime}=\vec{A}+\vec{\nabla} \lambda$ such that $\int \vec{A}^{\prime} d^{3} \vec{r}=\int \vec{\nabla} \lambda d^{3} \vec{r}$ $\neq 0$.

[15] J. M. Blatt and V. F. Weisskopf, Theoretical Nuclear Physics (Wiley, New York, 1952), p. 529. 